\title{
Analyses of the association between sialolithiasis and smoking, alcohol drinking and obesity: A nested case-control study using national health screening in Korean adults
}

\author{
Young Ju Jin \\ Wonkwang University College of Medicine \\ Young Eun Han \\ Seoul National University College of Medicine \\ Hyo Geun Choi ( $\square$ pupen@naver.com ) \\ Hallym University Sacred Heart Hospital https://orcid.org/0000-0003-1655-9549
}

\section{Research article}

Keywords: Sialolithiasis, Smoker, Alcohol, Obesity, Nested case-control study

Posted Date: March 25th, 2020

DOI: https://doi.org/10.21203/rs.2.20833/v2

License: (c) (1) This work is licensed under a Creative Commons Attribution 4.0 International License.

Read Full License

Version of Record: A version of this preprint was published at BMC Public Health on April 17th, 2020. See the published version at https://doi.org/10.1186/s12889-020-08674-w. 


\section{Abstract}

Background: Smoking and alcohol consumption are the most common social habits in patients with sialolithiasis. Moreover, obesity has been reported to have a significant association with poor oral hygiene, one of the causes of sialolithiasis. The purpose of this study was to evaluate the relationships among tobacco smoking, drinking alcohol, obesity and sialolithiasis in a Korean population. Methods: The Korean National Health Insurance Service-Health Screening Cohort, which includes patients $\geq 40$ years old, was assessed from 2002 to 2013. A total of 947 sialolithiasis participants were matched with 3,788 control subjects at a ratio of 1:4 with respect to age group, sex, income group, region of residence, hypertension, diabetes, and dyslipidemia. We analyzed the participants' previous histories of smoking (current or past smokers compared to nonsmokers) and alcohol consumption ( $\geq 1$ time per week compared to $<1$ time per week) in the sialolithiasis and control groups. Obesity was measured using body mass index (BMl, $\mathrm{kg} / \mathrm{m} 2$ ), which was categorized as $<18.5$ (underweight), $\geq 18.5$ and $<23$ (normal), $\geq 23$ and $<25$ (overweight), $\geq 25$ and $<30$ (obese I), and $\geq 30$ (obese II). Crude and adjusted odds ratios (ORs) and 95\% confidence intervals (Cls) were calculated using conditional logistic regression analyses. Results: The rate of smoking was higher in the sialolithiasis group (32.4\% [307/947]) than in the control group $(29.1 \%[1,103 / 3,788], P=0.047)$. The adjusted OR of smoking for the sialolithiasis group was $1.31(95 \% \mathrm{Cl}=1.08-1.59, \mathrm{P}=0.006)$. Alcohol consumption and obesity were not statistically significantly related to sialolithiasis. Conclusion: The odds of smoking were increased in sialolithiasis patients compared with control subjects in the population $\geq 40$ years of age.

\section{Background}

Sialolithiasis, which is the presence of a salivary duct stone, is the most common cause of major salivary gland swelling and pain in the ipsilateral salivary gland, typically when eating $[1,2]$. The annual incidence of sialolithiasis was 5.5/100,000 in Denmark in 2015 [3]; 2.7-5.9/100,000 in England in 1999 and 2000 [4, 5], based on hospital data; and 1.4-2.3/100,000 in Taiwan from 1996 to 2013, based on a health insurance research database [6]. The incidence rate increased to 7.3-14.1/100,000 from 2.05$3.98 / 100,000$ in children (those younger than 20 years old) in a Danish population-based study [7]. However, there are no reports on the incidence rate of sialolithiasis in Korea. The submandibular glands are the most commonly affected glands ( $80 \%-90 \%)$, followed by the parotid glands $(5 \%-20 \%)$ and the sublingual glands (very rare) $[1,2,8]$. The predominant prevalence in the submandibular gland occurs because Wharton's duct has a longer course and a larger diameter than Stenson's duct; thus, saliva flows against gravity and has a more viscous composition with a higher calcium and mucin content in this gland than in other salivary glands $[9,10]$.

The exact pathogenesis of stone formation in the salivary gland remains unclear. It is generally accepted that reduced saliva flow leads to an accumulation of salivary stones [11]. The retrograde theory suggests that food substances or bacteria in the oral cavity may ascend the salivary ducts and act as a nidus for further calcification [12]. 
Smoking and alcohol consumption are the most common social habits in patients with sialolithiasis. The association between sialolithiasis and smoking has been reported in several previous studies $[1,10,11$, $13,14]$. However, evidence of an association between alcohol consumption and sialolithiasis is very rare even though alcohol drinking is one of the most common causes of oral cavity and deep neck infection $[15,16]$. Obesity has been reported as a risk factor for various kinds of inflammatory disease, including periodontal disease [17]. In addition, previous studies have reported that poor oral health behaviors showed positive correlation with obesity $[18,19]$. Therefore, we hypothesized that obesity could be related to sialolithiasis caused by an oral infection that spreads to a salivary duct. The aim of this study was to evaluate the association between tobacco smoking, alcohol consumption, and obesity and sialolithiasis in a nationwide population-based cohort study using data from the Korean National Health Insurance Service-Health Screening Cohort (NHIS-HEALS). In this study, we estimated the odds ratios (ORs) of smoking, alcohol consumption and obesity in sialolithiasis patients compared to participants in a 1:4 matched control group.

\section{Materials And Methods}

\section{Study population and data collection}

This national cohort study relied on data from the Korean NHIS-HEALS [20-23]. The Korean NHIS selects random samples of $\sim 10 \%(n=\sim 515,000)$ directly from the entire population that underwent health evaluations from 2002 through $2013(n=\sim 5,150,000)$. Age- and sex-specific distributions of the cohort population have been described online [24]. The details of the methods used to perform these procedures are provided by the National Health Insurance Sharing Service [25].

All insured Koreans who are at least 40 years old and their dependents undergo no-cost biannual health evaluations. Each examinee must complete a standard questionnaire for this health evaluation program [26]. Because all Korean citizens are recognized by a 13-digit resident registration number from birth to death, exact population statistics can be determined using this database. It is mandatory for all Koreans to enroll in the NHIS. All Korean hospitals and clinics use the 13-digit resident registration number to register individual patients in the medical insurance system. Therefore, the risk of overlapping medical records is minimal, even if a patient moves from one place to another. Moreover, all medical treatments in Korea can be tracked without exception using the Korean Health Insurance Review \& Assessment (HIRA) system. In Korea, it is legally required to provide notice of death to an administrative entity before the funeral and the causes and date of death are recorded by medical doctors on a death certificate.

This cohort database included (i) personal information, (ii) health insurance claim codes (procedures and prescriptions), (iii) diagnostic codes based on the International Classification of Disease-10 (ICD-10), (iv) death records from the Korean National Statistical Office (using the Korean Standard Classification of disease), (v) socioeconomic data (residence and income), (vi) medical examination data, and (vii) health check-up data (body mass index [BMI], alcohol consumption, smoking habits, blood pressure, urinalysis, 
hemoglobin, fasting glucose, lipid parameters, creatinine, and liver enzymes) for each participant for the period from 2002 to $2013[25,26]$.

\section{Participant selection}

Out of 514,866 cases with 497,931,549 medical claim codes, we included participants who were diagnosed with sialolithiasis $(n=1,037)$. The sialolithiasis participants were matched $1: 4$ with participants among this cohort who were never diagnosed with sialolithiasis from 2002 through 2013 (control group). The control group was selected from the original population $(n=513,829)$. Subjects were matched by age group, sex, income group, region of residence, and medical history (e.g., hypertension, diabetes, and dyslipidemia). Participants in the control group were sorted using a random number order and selected from top to bottom to prevent selection bias. It was assumed that the matched control participants were involved at the same time as each matched participant with sialolithiasis (index date). Therefore, participants in the control group who died before the index date were excluded. Participants with sialolithiasis who had no history of health evaluations before the index date were excluded $(n=89)$. One participant with sialolithiasis was excluded due to the lack of a matching participant. Finally, 1:4 matching resulted in the inclusion of 947 participants with sialolithiasis and 3,788 control participants. We analyzed the previous health evaluation data in the sialolithiasis and control groups after matching (Fig. 1). In this study, we used the most recent health evaluation data before the index date.

\section{Variables}

\section{Independent variables}

Tobacco smoking was categorized based on the current smoking status (nonsmoker or past smoker/current smoker), duration of smoking (nonsmoker, $<20$ years, and $\geq 20$ years), and current number of cigarettes smoked per day ( 0 cigarettes per day, $<20$ cigarettes per day, and $\geq 20$ cigarettes per day, S1 Table). We selected the current smoking status in this study. Current and past smokers were defined as smokers and were compared to nonsmokers.

Alcohol consumption was evaluated by frequency ( $<1$ time per week, and $\geq 1$ time per week) and by the amount of alcohol consumed at a time ( $<1$ soju bottle, 1 soju bottle, and $>1$ soju bottle, S1 Table). Generally, a bottle of soju contains $17.5 \%$ of alcohol per $360 \mathrm{ml}$. A bottle of soju is equivalent to approximately 3.5 bottles of beer. We selected the frequency of alcohol consumption in this study. We used alcohol consumption to define alcohol consumption $\geq 1$ time per week compared to alcohol consumption $<1$ time per week.

Obesity was measured using BMI $\left(\mathrm{kg} / \mathrm{m}^{2}\right)$ and was categorized as $<18.5$ (underweight), $\geq 18.5$ and $<23$ (normal), $\geq 23$ and $<25$ (overweight), $\geq 25$ and $<30$ (obese I), and $\geq 30$ (obese II) following the WPRO 2000 guidelines [27].

\section{Covariate analysis}


The age groups were classified using 5-year age intervals: $40-44,45-49,50-54, \ldots$, and $85+$ years old. A total of 10 age groups were designated. Income was initially divided into 41 classes according to health care premium (one health assistance class, 20 self-employment health insurance classes, and 20 employment health insurance classes). These groups were recategorized into 5 classes (classes 1 [lowest income]-5 [highest income]). The region of residence was divided into 16 areas according to administrative district. These regions were regrouped into urban (Seoul, Busan, Daegu, Incheon, Gwangju, Daejeon, and Ulsan) and rural (Gyeonggi, Gangwon, Chungcheongbuk, Chungcheongnam, Jeollabuk, Jeollanam, Gyeongsangbuk, Gyeongsangnam, and Jeju) areas.

The participants' prior medical histories were evaluated using ICD-10 codes. To ensure an accurate diagnosis, hypertension (I10 and I15), diabetes (E10-E14), and dyslipidemia (E78) were regarded as present if a participant was treated $\geq 2$ times.

\section{Dependent variable}

Sialolithiasis was diagnosed based on the ICD-10 code K115.

\section{Statistical Analyses}

Chi-square tests were used to compare the general characteristics of the sialolithiasis and control groups.

To analyze the ORs of smoking, drinking alcohol, and obesity in sialolithiasis patients, conditional logistic regression analysis was used. In this analysis, a crude (simple) adjusted model (adjusted for obesity, smoking status, and frequency of alcohol consumption) was used, and $95 \%$ confidence intervals (Cls) were calculated. In these analyses, age group, sex, income group, region of residence, hypertension, diabetes, and dyslipidemia were stratified.

For the subgroup analyses, we divided the participants by age and sex ( $<60$ years, $\geq 60$ years; male and female). The division of the age groups was determined by the median age of all the participants.

Two-tailed analyses were conducted, and $P$ values less than 0.05 were considered to indicate significance. The results were statistically analyzed using SPSS v. 22.0 (IBM, Armonk, NY, USA).

\section{Results}

The rate of tobacco smoking was higher in the sialolithiasis group (32.4\% [307/947]) than in the control group $(29.1 \%$ [1,103/3,788], $P=0.047$, Table 1$)$. Alcohol consumption and obesity were not different between the sialolithiasis and control groups. The general characteristics (age, sex, income, region of residence, hypertension, diabetes, and dyslipidemia) of the participants were the same due to the matching procedure $(P=1.000)$.

The adjusted OR for smoking in the sialolithiasis group was $1.31(95 \% \mathrm{Cl}=1.08-1.59, \mathrm{P}=0.006$, Table 2). The adjusted ORs for alcohol consumption and obesity in the sialolithiasis group did not reach statistical 
significance. The adjusted OR for alcohol consumption in the sialolithiasis group was $0.86(95 \% \mathrm{Cl}=$ $0.71-1.04, P=0.119)$. The adjusted ORs for obesity in the sialolithiasis group were $0.56(95 \% \mathrm{Cl}=0.31$ $1.01, \mathrm{P}=0.055)$ for $\mathrm{BMI}<18.5 ; 1.00$ for $\mathrm{BMI} \geq 18.5$ to $<23 ; 1.09(95 \% \mathrm{Cl}=0.90-1.31, \mathrm{P}=0.376)$ for $\mathrm{BMI} \geq$ 23 to $<25 ; 0.98(95 \% \mathrm{Cl}=0.81-1.17, \mathrm{P}=0.800)$ for $\mathrm{BMI} \geq 25$ to $<30$; and $1.36(95 \% \mathrm{Cl}=0.93-1.98, \mathrm{P}=$ $0.119)$ for $\mathrm{BMI} \geq 30$.

In subgroup analyses performed according to age and sex, the adjusted OR for smoking with statistical significance was 1.36 and 1.27 for participants $<60$ years old $(95 \% \mathrm{Cl}=1.06-1.75, \mathrm{P}=0.017)$ and for men $(95 \% \mathrm{Cl}=1.03-1.56, \mathrm{P}=0.023$, Table 3$)$, respectively. The crude and adjusted ORs for drinking alcohol and obesity were not statistically significant for either sex and all age groups. The rates of smoking, alcohol consumption, and obesity were evaluated according to age groups ( $<60$ years vs. $\geq 60$ years) and sex (male vs. female). The rates were significantly different among all the groups (S2).

Additionally, the association between smoking duration and sialolithiasis was evaluated. The adjusted ORs for the association between smoking and sialolithiasis were $1.34(95 \% \mathrm{Cl}=1.08-1.66, \mathrm{P}=0.007$ among those with a duration of smoking $\geq 20$ years and $1.24(95 \% \mathrm{Cl}=0.94-1.63, \mathrm{P}=0.123$, S3 table) among those with a duration of smoking $<20$ years.

\section{Discussion}

In our study, the rate of smoking was higher in the sialolithiasis group (32.4\%) than in the control group (29.1\%), and the adjusted OR of smoking was significantly higher in the sialolithiasis group than in the control group (adjusted $\mathrm{OR}=1.31,95 \% \mathrm{Cl}=1.08-1.59$ ). However, the rates of alcohol consumption and obesity did not differ between the sialolithiasis and control groups. We found the similar results when we analyzed these factors according to the duration of smoking and the amount of alcohol consumed. In particular, longer periods of smoking were significantly related to sialolithiasis. Similar to our findings, a history of smoking has been reported to be a predisposing factor for sialolithiasis in the general population (adjusted $\mathrm{OR}=1.21,95 \% \mathrm{Cl}=1.02-1.44, \mathrm{P}=0.028$ ) [28]. Stone sizes have been reported to be much larger in current smokers than in ex-smokers (12.4 mm vs. $7.5 \mathrm{~mm}, \mathrm{P}=0.03)$ [13].

Tobacco smoking can cause oral cavity inflammation [29]. Because the salivary duct opens to the oral cavity, inflammation in the oral cavity can be transmitted into the salivary duct and cause ductal swelling and narrowing. Decreased salivary duct diameter can induce salivary stasis and cause the precipitation of salivary stones. Salivary stones and ductal inflammation increase the occurrence of sialolithiasis. After the formation of salivary duct stones, bacteria can ascend the duct more easily from the mouth and proliferate on the surface of the stones, contributing to the further development of sialolithiasis [11]. Moreover, long-term smoking decreases salivary flow and thus decreases antimicrobial proteins, including IgA, a-amylase and lysozyme, which are important for oral mucosal immunity [30, 31]. Therefore, a vicious cycle that accelerates the formation of salivary gland stones ensues.

In this study, alcohol consumption and obesity did not differ between the sialolithiasis and control groups. Similar to tobacco smoking, alcohol consumption and obesity play an important role in oral 
health. There was a study that found no significant difference in alcohol consumption between the 50 cases with salivary gland stones and the 90 cases without stones ( $P=0.597)$ [32] \{Kraaij, $2015 \# 22\}$. Because sialolithiasis can be promoted by oral cavity inflammation, we presumed that alcohol intake might be associated with sialolithiasis. Generally, the association between alcohol consumption and inflammation is known to depend on the amount of alcohol consumed. The influence of low to moderate alcohol intake on health outcomes is controversial; however, excessive alcohol intake is associated with worse outcomes [33]. In particular, light to moderate drinking has shown protective effects against cardiovascular disease, and this amount has been defined as daily consumption of a 5 to 6 oz glass of red wine [34]. In our data, we did not compare the amounts of alcohol consumed; instead, we examined the frequency of alcohol consumption ( $<1$ time per week, $\geq 1$ time per week). Moreover, the small number of participants might not have been enough to reach statistical significance.

Obesity is known to be a state of abnormal immune activity and increased susceptibility to inflammatory diseases, such as periodontal disease, diabetes, airway inflammation, cardiovascular disease and fatty liver disease $[17,35]$. Therefore, we hypothesized that obesity might also be related to sialolithiasis. However, a comparison among the 5 groups divided by BMI did not show any difference. Additional analyses of these groups according to sex and age did not show any differences. There are no previous reports regarding obesity and sialolithiasis. One study reported that the salivary flow is not decreased in obese patients compared to nonobese patients. However, the activity of salivary enzymes, such as lipase and a-amylase, which break down nutrients, was much higher in the obese group [36]. Therefore, obesity can be considered the cause of chronic inflammation, which can lead to the formation of salivary stone, but a normal amount of salivary flow and enzymes with high activity can interfere with the salivary stone formation.

This study has several benefits. In contrast to hospital-based studies, we used participants from a large representative nationwide population who had undergone health screening examinations. We analyzed the ORs for tobacco smoking, alcohol consumption and obesity based on BMI in the sialolithiasis group and compared them to the ORs in a well-matched control group. The control group was randomly selected and matched by age group, sex, income group, region of residence, and medical history (e.g., hypertension, diabetes, and dyslipidemia) to prevent selection bias. Moreover, we used an adjusted logistic regression model to minimize confounders.

This study has several limitations. First, we used patient claim codes from the HIRA database to confirm which individuals were diagnosed with sialolithiasis. These data may indicate the incidence of sialolithiasis. Second, the participants self-reported their health status and habits, and self-reporting can be biased. Third, we could not evaluate the amount of alcohol consumed, which has an important effect on oral health. Fourth, we did not have data from patients who were younger than 40 years old because the health screening examinations were performed in individuals older than 40 years. Fifth, the number of participants with sialolithiasis was relatively small because of the low incidence rate of this disease. Sixth, the rate of smoking was statistically significant different between the sialolithiasis and control groups, but the difference was not large. Finally, we did not observe an association between female sex 
and smoking. The low rates of smoking in women compared to men might have affected this finding (S2 Table).

\section{Conclusion}

Tobacco smoking showed a positive association with sialolithiasis in the population $\geq 40$ years old. However, the association of alcohol consumption and obesity with sialolithiasis did not reach statistical significance.

\section{List Of Abbreviations}

OR: odds ratio; Cl: confidence interval; NHIS-HEALS: National Health Insurance Service-Health Screening Cohort; HIRA: Health Insurance Review and Assessment; BMI: body mass index; WPRO: Regional Office for the Western Pacific

\section{Declarations}

\section{Ethics approval and consent to participate:}

The Ethics Committee of Hallym University (2017-I102) approved the use of these data. The study was exempted from the need for written informed consent by the institutional review board.

\section{Consent for publication}

Not applicable.

\section{Availability of data and materials:}

The datasets analyzed during the current study are in the public domain and are freely available from http://nhiss.nhis.or.kr/ with permission.

\section{Competing interests}

The authors declare that they have no competing interests

\section{Funding}

This work was supported in part by a research grant (NRF-2018-R1D1A1A0-2085328 and NRF-2019R1G1A1-099842) from the National Research Foundation (NRF) of Korea.

\section{Author' contributions}

Jin $Y J$ and Han YE wrote the manuscript. Jin YJ and Choi HG acquired the data and performed the analysis. Jin YJ, Han YE and Choi HG edited the manuscript. All authors read and approved the final 
manuscript.

\section{Acknowledgments}

Contributors: The manuscript was edited for English language, grammar, punctuation, spelling, and overall style by the highly qualified native English-speaking editors at American Journal Experts (FAOBDB3C-9A90-29F6-DBCP).

\section{References}

1. Huoh KC, Eisele DW. Etiologic factors in sialolithiasis. Otolaryngology--Head and Neck Surgery. 2011;145:935-939.

2. Sigismund PE, Zenk J, Koch M, Schapher M, Rudes M, Iro H. Nearly 3,000 salivary stones: some clinical and epidemiologic aspects. The Laryngoscope. 2015;125:1879-1882.

3. Schrøder S, Homøe P, Wagner N, Vataire A-L, Madsen HEL, Bardow A. Does drinking water influence hospital-admitted sialolithiasis on an epidemiological level in Denmark? BMJ open. 2015;5:e007385.

4. Escudier M, McGurk M. Economics: Symptomatic sialoadenitis and sialolithiasis in the English population, an estimate of the cost of hospital treatment. British dental journal. 1999;186:463.

5. Sherman J, McGurk M. Lack of correlation between water hardness and salivary calculi in England. British Journal of Oral and Maxillofacial Surgery. 2000;38:50-53.

6. Wang $\mathrm{Y}-\mathrm{H}$, Chen $\mathrm{Y}-\mathrm{T}$, Chiu Y-W, Yu H-C, Chang Y-C. Time trends in the prevalence of diagnosed sialolithiasis from Taiwanese nationwide health insurance dental dataset. Journal of Dental Sciences. 2019;14:365-369.

7. Schrøder SA, Andersson M, Wohlfahrt J, Wagner N, Bardow A, Homøe P. Incidence of sialolithiasis in Denmark: a nationwide population-based register study. European Archives of Oto-RhinoLaryngology. 2017;274:1975-1981.

8. Lustmann J, Regev E, Melamed Y. Sialolithiasis: a survey on 245 patients and a review of the literature. International journal of oral and maxillofacial surgery. 1990; 19:135-138.

9. Rogers J, McCaffrey T. Inflammatory disorders of the salivary glands. Otolaryngology: Head \& Neck Surgery 5th ed Philadelphia, Pa: Mosby Elsevier 2010.

10. Williams MF. SIALOLITHIASIS. Otolaryngologic Clinics of North America, 1999; 32:819-834.

11. Harrison JD. Causes, natural history, and incidence of salivary stones and obstructions. Otolaryngologic clinics of North America. 2009;42:927-947.

12. Marchal F, Kurt A-M, Dulguerov P, Lehmann W. Retrograde theory in sialolithiasis formation. Archives of Otolaryngology-Head \& Neck Surgery. 2001;127:66-68.

13. Wu C-C, Hung S-H, Lin H-C, Lee C-Z, Lee H-C, Chung S-D. Sialolithiasis is associated with nephrolithiasis: a case-control study. Acta oto-laryngologica. 2016;136:497-500.

14. Yiu A, Kalejaiye A, Amdur R, Hesham HT, Bandyopadhyay B. Association of serum electrolytes and smoking with salivary gland stone formation. International journal of oral and maxillofacial surgery. 
2016;45:764-768.

15. Hung SH, Huang HM, Lee HC, Ching Lin H, Kao LT, Wu CS. A population-based study on the association between chronic periodontitis and sialolithiasis. The Laryngoscope. 2016;126:847-850.

16. Khairnar M, Wadgave U, Khairnar S. Effect of Alcoholism on Oral Health: A Review. J Alcohol Drug Depend. 2017;5:3.

17. Morita I, Okamoto Y, Yoshii S, Nakagaki H, Mizuno K, Sheiham A et al. Five-year incidence of periodontal disease is related to body mass index. J Dent Res. 2011;90: 199-202.

18. de Oliveira C, Watt R, Hamer M. Toothbrushing, inflammation, and risk of cardiovascular disease: results from Scottish Health Survey. Bmj. 2010;340:c2451.

19. Park JB, Nam GE, Han K, Ko Y, Park YG. Obesity in relation to oral health behaviors: An analysis of the Korea National Health and Nutrition Examination Survey 2008-2010. Experimental and therapeutic medicine. 2016;12:3093-3100.

20. Lee J, Lee JS, Park S-H, Shin SA, Kim K. Cohort profile: The national health insurance servicenational sample cohort (NHIS-NSC), South Korea. International journal of epidemiology. 2016;46:e15e15.

21. Seong SC, Kim YY, Park SK, Khang YH, Kim HC, Park JH et al. Cohort profile: the national health insurance service-national health screening cohort (NHIS-HEALS) in Korea. BMJ open. 2017;7: e016640.

22. Shin WY, Lee T, Jeon DH, Kim HC. Diabetes, frequency of exercise, and mortality over 12 years: Analysis of the National Health Insurance Service-Health Screening (NHIS-HEALS) Database. Journal of Korean medical science. 2018;33.

23. Lee JW, You NY, Kim Y, Kim Y, Kim J, Kang HT. Statin use and site-specific risk of colorectal cancer in individuals with hypercholesterolemia from the National Health Insurance Service-National Health Screening Cohort (NHIS-HEALS). Nutrition, Metabolism and Cardiovascular Diseases. 2019;29:701709.

24. Statistics Korea. http://kosis.kr/eng/statisticsList/statisticsList 01List.jsp? vwcd=MT_ETITLE\&parentld=B\#SubCont (accessed date July 24, 2017).

25. http://nhiss.nhis.or.kr/ (accessed date June 18, 2017).

26. Song SO, Jung $\mathrm{CH}$, Song YDet al. Background and data configuration process of a nationwide population-based study using the korean national health insurance system. Diabetes \& metabolism journal. 2014;38:395-403.

27. World Health Organization. The Asia-Pacific perspective: redefining obesity and its treatment. Sydney: Health Communications Australia. 2000.

28. Hung SH, Lin HC, Su CH, Chung SD. Association of sialolithiasis with cholelithiasis: A populationbased study. Head \& neck. 2016;38:560-563.

29. Sheiham A, Watt RG. The common risk factor approach: a rational basis for promoting oral health. Community Dentistry and Oral Epidemiology: Commentary. 2000;28:399-406. 
30. Rad M, Kakoie S, Brojeni FN, Pourdamghan N. Effect of long-term smoking on whole-mouth salivary flow rate and oral health. Journal of dental research, dental clinics, dental prospects. 2010;4:110.

31. Fortes MB, Diment BC, Di Felice U, Walsh NP. Dehydration decreases saliva antimicrobial proteins important for mucosal immunity. Applied Physiology, Nutrition, and Metabolism. 2012;37:850-859.

32. 32. Kraaij S, Karagozoglu KH, Kenter YA, Pijpe J, Gilijamse M, Brand HS. Systemic diseases and the risk of developing salivary stones: a case control study. Oral surgery, oral medicine, oral pathology and oral radiology. 2015;119:539-543.

33. Goral J, Karavitis J, Kovacs EJ. Exposure-dependent effects of ethanol on the innate immune system. Alcohol. 2008;42:237-247.

34. O'Keefe JH, Bhatti SK, Bajwa A, DiNicolantonio JJ, Lavie CJ. Alcohol and cardiovascular health: the dose makes the poison... or the remedy Mayo Clinic Proceedings: Elsevier. 2014:382-393.

35. Wellen KE, Hotamisligil GS. Inflammation, stress, and diabetes. The Journal of clinical investigation. 2005;115:1111-1119.

36. Mennella I, Fogliano V, Vitaglione P. Salivary lipase and a-amylase activities are higher in overweight than in normal weight subjects: Influences on dietary behavior. Food research international. 2014;66:463-468.

\section{Figures}


514,866 Participants with 497,931,549 Medical claim codes

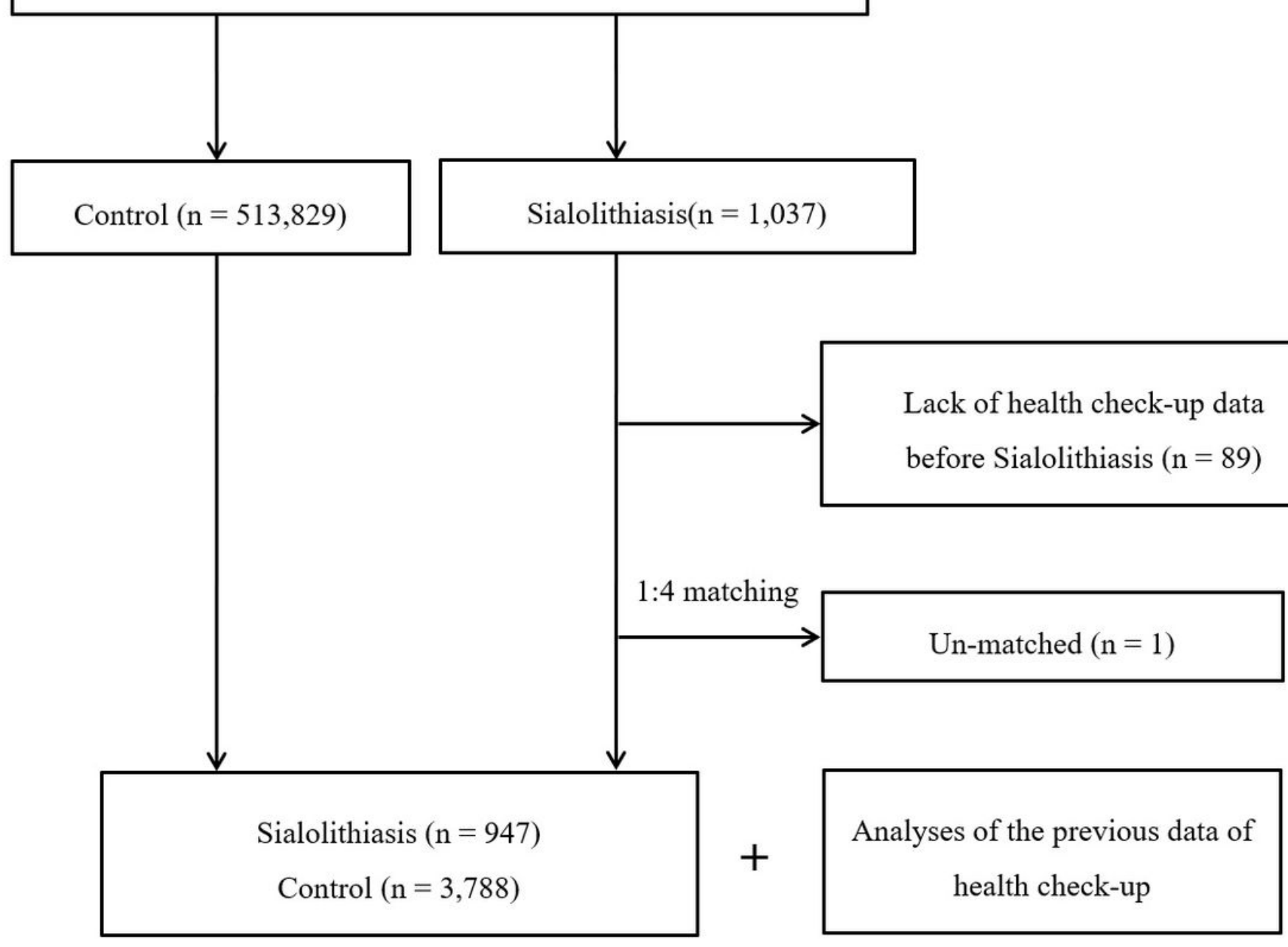

\section{Figure 1}

Schematic illustration of the participant selection process used in the present study. Of a total of 514,866 participants, 947 sialolithiasis patients were matched with 3,788 control participants by age, group, sex, income group, region of residence, and past medical history.

\section{Supplementary Files}

This is a list of supplementary files associated with this preprint. Click to download.

- TableSupplement.docx.pdf 